\title{
Endonasal choanal atresia repair; evaluating the surgical results of 58 cases
}

\author{
Münir Demir Bajin ${ }^{1 \oplus}$, Övsen Önay ${ }^{2 \oplus}$, Rıza Önder Günaydın ${ }^{1 \oplus}$, Ömer Faruk Ünal ${ }^{3 \oplus}$, \\ Ömer Taşkın Yücel ${ }^{1 \oplus}, \mathrm{Umut} \mathrm{Akyol}^{1 \oplus}, \mathrm{Canset} \mathrm{Aydın}^{4 \oplus}$
}

${ }^{1}$ Department of Otorhinolaryngology, Hacettepe University Faculty of Medicine, ${ }^{2}$ Department of Otorhinolaryngology, Attlim University Faculty of Medicine, Ankara; ${ }^{3}$ Department of Otorhinolaryngology, Koç University Faculty of Medicine, İstanbul; ${ }^{4}$ Clinic of Otorhinolaryngology, Medicana International Ankara Hospital, Ankara, Turkey.

\begin{abstract}
Background. Choanal atresia is the most common congenital nasal anomaly, with an incidence of 1:5000-1:8000 live births. Atresia can be seen as membraneous, bony or mixed type. When it is bilateral, it is accepted lifethreatening, therefore bilateral atresia necessitates immediate intervention. Diagnosis is confirmed by endoscopic examination and computed tomography. The absolute treatment is surgical, and different approaches have been proposed.

Methods. Herein, we describe our 15-year experience in the treatment of 58 patients of congenital choanal atresia with transnasal endoscopic approach, and we compare the efficacy of placement of an intranasal stent and applying mitomycin while endoscopic microsurgical repair.

Results. The study included 41 female patients $(71 \%)$ and 17 male patients $(29 \%)$ with congenital CA. The mean age was 3 years ranging from 10 days to 16 years. The atretic plate was bilateral in 24 patients $(41 \%)$ and unilateral in $34(59 \%)$. The most common atresia type was the mixed type with 29 patients $(50 \%)$. A total of 17 patients (29\%) required postoperative revision(s). Postoperative revisions were more frequent among patients with bilateral CA $(50 \%)$, and with mixed CA $(31 \%)$. Stenting was used additionally by surgical correction for 10 patients. After stenting, fibrosis and restenosis was seen in 7 patients $(79 \%)$. Mitomycin C was applied peroperatively in 8 patients. Restenosis after mitomycin application was seen in 4 patients (50\%).

Conclusions. By our experience, endoscopic microsurgical repair of atresia proved to be an effective and safe procedure, results compared with adjuvant treatment modalities like stent or mitomycin $\mathrm{C}$ use, was not better. Restenosis was the major problem seen after surgical correction.
\end{abstract}

Key words: choanal atresia, stent, transnasal endoscopic approach, mitomycin C.

Choanal atresia (CA) is the most common congenital nasal anomaly, and is encountered in approximately 1:5000-8000 live births and it is slightly more prevalent among females. ${ }^{1,2}$ The failure of the embryologic nasobuccal membrane of Hochstetter to rupture gives rise to $\mathrm{CA} .{ }^{3}$ As a result of the unsuccessful membranous rupture, the nasal cavity is narrowed by a bony obstruction of the choanae

$凶$ Övsen Önay

ovsenonay8@gmail.com

Received 2nd August 2019, revised 4th February 2020, 24th March 2020, accepted 6th April 2020. which is caused by the pterygoid plate laterally and vomer medially. ${ }^{3}$ Most cases also have a mucosal membranous component to the obstruction. More than half of the patients $(70 \%)$ have a mixed bony-membranous atresia. ${ }^{3}$ CA may be unilateral (more common, $60 \%$ of all cases) or bilateral. ${ }^{4}$ Owing to the necessity of nasal breathing in newborns, bilateral cases present as an airway emergency existing with respiratory distress since the moment of birth. Bilateral atresia patients characteristically present with cyclic cyanotic spells that improve with crying (paradoxical cyanosis). ${ }^{5}$ Initial therapy of this airway obstruction, paradoxical 
cyanosis, and stridor; should concern immediate maintenance of an adequate airway by oral airway or intubation. ${ }^{5}$ Unilateral CA presents usually between 5 and 24 months, with a snoring cry, unilateral rhinorrhea, mouth breathing, respiratory failure and may go unnoticed. ${ }^{5,6}$ When there is a suspicion of CA, it can be evaluated by advancing a nasogastric tube through the nostrils or searching the presence/ absence of airflow out of the nostrils, and it can be directly confirmed by nasal endoscopic examination and computed tomography scan (CT). ${ }^{6}$ When nasal endoscopy is performed suboptimally, the definitive diagnosis is established with a CT scan of the paranasal sinuses and skull base. The narrowing of the posterior nasal cavity will be demonstrated by CT scan, such as medial bowing of the lateral wall of the nasal cavity, and the impingement at the level of the anterior aspect of the pterygoid plates and a widening of the posterior portion of the vomer. ${ }^{7}$ Patients with unilateral or bilateral CA should be screened for associated congenital disorders. Associated congenital anomalies may be identified in patients with CA, such as developmental delay, hormonal disorders, maxillofacial anomalies, cardiac disorders cerebral abnormalities. ${ }^{8}$

Almost all cases are now repaired via an endoscopic approach with the aid of powered instruments. ${ }^{3}$ Despite that, revision surgery may even be required, because the major problem encountered in the management of CA is re-stenosis after surgical correction, and it occurs because of scar and/or granulation tissue formation. Adjunct therapies and techniques have been employed to avoid such problems. Inserting nasal stents and applying topical mitomycin (an aminoglycoside antibiotic isolated from Streptomyces caespitosus) intraoperatively to the choanae can be performed to decrease the risk of re-stenosis, but the reported efficacy of these measures vary widely with some studies showing counterproductive effects. ${ }^{9}$

Herein, we describe our experience in the transnasal treatment of congenital CA with endoscopic approach efficacy of placement of an intranasal stent, and applying mitomycin in our tertiary care center.

\section{Material and Methods}

Institutional review board approval was obtained from Hacettepe University (GO 14-474, 5.11. 2014). Patient records, undergoing surgery for congenital CA at the Hacettepe University Faculty of Medicine's Otorhinolaryngology clinic over a period of 15 years (January 2000-January 2015) were evaluated retrospectively. Data collection was based on reviewing case histories. Eight patients who had correction surgery previously for CA at other institutions were excluded from the study. A total of 58 patients were analyzed in our study.

The variables analyzed; Gender, type of atretic plate, side of atresia, details of surgeries performed (transnasal endoscopic repair only or application of nasal stent or applying topical mitomycin C), the need of revision surgery were evaluated.

Preoperative evaluation; We performed a thorough prior assessment of all patients by nasal endoscopic examination, with a flexible fiberscope $(2.2 \mathrm{~mm})$ or a rigid endoscope (2.7 $\mathrm{mm})$, and for 13 patients we performed an additional CT scan of the craniofacial complex.

Surgical approach; All cases were performed by a transnasal approach using endoscopy under general anesthesia. We did not use navigation systems in any of the cases. In all cases, cottonoids impregnated in local anesthetic (lidocain) and a vasoconstrictor (adrenaline $1 \%$ were applied in both nasal fossa. Nasal endoscopy was then carried out using a nasal endoscope (2,8 mm, Karl Storz). Following the bottom of the nasal fossa, the choana was opened medially and inferiorly. The incision of the mucosa was carried out with sicle knife. Once the nasal mucosa covering the atretic plate was removed, the plate and the posterior part of the vomer were extracted with microsurgical 
instrumentation (ear curette or drill). The edges of the neo-choana were smoothed and the bone surface was covered with mucosa. All patients were treated with anti-reflux therapy postoperatively, and also antibiotics for 5 to 7 days (ampicillin+sulbactam $50 \mathrm{mg} / \mathrm{kg} /$ day). For 7 days, patients were fed through an orogastric probe. In addition, local postoperative treatment with nasal humidification and Daily suction was performed.

\section{Results}

The study included 58 patient with congenital CA. Patient characteristics are detailed in Table I. All cases were repaired via endoscopic approach, and the mean follow-up time after surgery was 51 months (ranging from 6 months to 8 years). As a support for the intranasal neochoana, $10(17 \%)$ patients underwent placement of a silicone stent (intubation tube, number of 3-4) for 1 to 4 weeks. Topical mitomycin C $(0.4 \mathrm{mg} / \mathrm{ml}$, for all rough surfaces, without using a stent) was used in $8(14 \%)$ patients at the time of when neo-choana was created. Of participants Transnasal endoscopic repair only was performed on 69\% (40), Transnasal endoscopic repair and Stenting was performed on $17 \%$ (10) and Transnasal endoscopic repair using Mitomycin C was performed on 14\% (8). The effectivity for surgery was determined by the absence of respiratory symptoms and patent choanae on endoscopic examination and no need for revision. A total of 17 (29\%) patients required postoperative revision, from 1 to 6 times. Revision surgery needs according to CA type and surgical correction type are detailed in Table II and III.

\section{Discussion}

$\mathrm{CA}$ is the most common congenital nasal anomaly. It is characterised by the obliteration of the posterior nasal opening, due to the imperforation of the oronasal membrane. The safety and efficacy of endoscopic repair of this imperforation has been well demonstrated., ${ }^{3,410}$ Postoperative formation of scar tissue, synechia and granulation tissue are commonly reported and all may be reasons for recurrent nasal obstruction. The use of adjuvant stenting and mitomycin $C$ has been adopted over the years in attempts to minimize these problems. Despite that, there is a controversy regarding the usefulness of placing stents, application of adjuvant mitomycin $\mathrm{C}^{4,9,11}$

Various possible techniques and surgical approaches were used to treat CA but currently two surgical approaches are mainly used: transnasal and transpalatal. The complications that can be seen as a result of the transpalatal approach are unusual but include, palatal flap breakdown or fistula, high arched palate deformity and need for orthodontic treatment, blood loss requiring transfusion. ${ }^{12}$ The main goal of surgery include establishing a normal nasal passage, and additionally it is essential to prevent further damage to the growing structures. ${ }^{13}$ By using transnasal endoscopic surgery approach, these goals can be achieved. Surgical repair with transnasal endoscopic technique provides an excellent visualisation of the posterior nasal defect and because of its safety and effectivity, transnasal endoscopic surgery has currently become the procedure of choice. ${ }^{14}$ Teissier et al. ${ }^{10}$ described the transnasal endoscopic repair as a well-tolerated technique, and despite of its learning curve, they concluded that it was a successfull treatment technique.

Table I. Patient characteristics.

\begin{tabular}{ll}
\hline Patient characteristics & $\mathrm{n}(\%)$ \\
\hline Gender & \\
$\quad$ Male & $17(29 \%)$ \\
$\quad$ Female & $41(71 \%)$ \\
Unilateral CA & $34(59 \%)$ \\
Right side & $14(24 \%)$ \\
Left side & $20(35 \%)$ \\
Bilateral CA & $24(41 \%)$ \\
Type of atresia & \\
$\quad$ Mixed & $29(50 \%)$ \\
$\quad$ Membranous & $16(28 \%)$ \\
$\quad$ Bony & $13(22 \%)$ \\
\hline
\end{tabular}

CA: choanal atresia 
Table II. Classification of revision surgery needs.

\begin{tabular}{ll}
\hline Revision surgery performed for & Number of revision surgery/Total number of defined patients (\%) \\
\hline Patients with unilateral CA & $5 / 34(15 \%)$ \\
Patients with bilateral CA & $12 / 24(50 \%)$ \\
Patients with bony CA & $4 / 13(30 \%)$ \\
Patients with membranous CA & $4 / 16(25 \%)$ \\
Patients with mixed CA & $9 / 29(31 \%)$ \\
\hline Total patients & $17 / 58(29 \%)$ \\
\hline
\end{tabular}

CA: choanal atresia

Table III. Revision need, after surgery classified according to type of management.

\begin{tabular}{ll}
\hline Surgical details for revision surgery & Number of revision surgery/Total number of defined patients (\%) \\
\hline $\begin{array}{l}\text { Revision needed after transnasal } \\
\text { endoscopic repair+ stenting }\end{array}$ & $7 / 10(70 \%)$ \\
$\begin{array}{l}\text { Revision needed after transnasal } \\
\text { endoscopic repair+ using Mitomycin C }\end{array}$ & $4 / 8(50 \%)$ \\
$\begin{array}{l}\text { Revision needed after transnasal } \\
\text { endoscopic repair only }\end{array}$ & $6 / 40(15 \%)$ \\
\hline Total revision patients & $17 / 58(29 \%)$ \\
\hline
\end{tabular}

José Luis Llorente et al. ${ }^{4}$ published their surgical results of 10 patients. Seven cases $(70 \%)$ with a bilateral CA and, 3 cases (30\%) with unilateral CA had been operated. They used transnasal endoscopic surgical repair in all cases. Neither stents nor topical mitomycin were used in any case. After a mean follow-up of 27 months, no restenosis were detected in any of the cases and no further surgery or dilatations were required, so they gave a $100 \%$ success rate with endoscopic repair.

Traditionally, the use of stents has been a part of the postoperative management of CA repair for maintaining the patency for newly formed choana. The use of stents for CA repair is very controversial in the relating literature. Some reports have found that stenting is associated with an increased incidence of restenosis up to $50-70 \% .{ }^{15}$ Stents may induce a foreign body reaction, and be an infectious nidus, which will trigger an inflammatory response. As a result of inflammation, it may lead to granulation tissue formation, and eventually restenosis. ${ }^{9}$ In our study, patients whom we inserted stents had higher incidence of restenosis 7/10 (70\%) than those who had no stent with a restenosis rate of $6 / 40(15 \%)$. More than half of the patients that used a stent needed revision, therefore stent usage was not seen successful for the correction of CA, and that was attributed to continuous inflammation caused by stents, the greater formation of granulation tissue and chronic inflammation resulting in fibrosis and restenosis.

Mitomycin C is a topical aminoglycoside antibiotic that is thought to reduce scar and granulation tissue formation by the inhibition of fibroblast growth and migration. ${ }^{3}$ The use of mitomycin $\mathrm{C}$, proposed reducing the scar formation and improvement in healing, and it had been used as an additional therapy for CA repair. The use of mitomycin in the larynx and trachea, improves the outcomes, but its efficacy in patients with $\mathrm{CA}$, in preventing restenosis has been variable. ${ }^{11,16,17}$ Holland et al. ${ }^{17}$ found an improvement in the outcome and increase in re-stenosis with the use of mitomycin, with a small number of patients. Following Holland et al. ${ }^{17}$, Prasad et al. ${ }^{18}$ also proposed of a lower incidence of re-stenosis, following these reports the use of mitomycin $C$ gained an increasing acceptance. Kubba et al. $^{5}$ and $\mathrm{Al}$-Ammar et 
al. ${ }^{19}$ did not find any significant difference in the outcome between patients who received mitomycin $C$ treatment and those who did not. Likewise, when assesing the long term success of mitomycin, Teissier et al. ${ }^{10}$ showed non persuasive effect. In our study, 50\% (n:4/8) of those who had mitomycin $C$ at primary surgery had at least one restenosis requiring revision surgery. Comparison between the efficacy of adjacent modality addited to surgery showed that Mitomiycin $C$ was more effective than stent usage in our experience, but the role of topical mitomycin C was not associated with any significant improvment either.

By our experience, endoscopic microsurgical repair of atresia proved to be an effective and safe procedure, results compared with adjuvant treatment modalities like stent or mitomycin C use, was not better. Restenosis was the major problem seen after surgical correction.

\section{REFERENCES}

1. Bedwell J, Shah RK, Bauman N, Zalzal GH, Preciado DA. Balloon dilation for management of choanal atresia and stenosis. Int J Pediatr Otorhinolaryngol 2011; 75: 1515-1518.

2. Corrales CE, Koltai PJ. Choanal atresia: current concepts and controversies. Curr Opin Otolaryngol Head Neck Surg 2009; 17: 466-470.

3. Carter JM, Lawlor C, Guarisco JL. The efficacy of mitomycin and stenting in choanal atresia repair: a 20 year experience. Int J Pediatr Otorhinolaryngol 2014; 78: 307-311.

4. Llorente JL, Lopez F, Morato M, Suarez V, Coca A, Suarez C. Endoscopic treatment of choanal atresia. Acta Otorrinolaringol Esp 2013; 64: 389-395.

5. Kubba H, Bennett A, Bailey CM. An update on choanal atresia surgery at Great Ormond Street Hospital for Children: preliminary results with Mitomycin C and the KTP laser. Int J Pediatr Otorhinolaryngol 2004; 68: 939-945.

6. Rodriguez H, Cuestas G, Passali D. A 20-year experience in microsurgical treatment of choanal atresia. Acta Otorrinolaringol Esp 2014; 65: 85-92.

7. Aslan S, Yilmazer C, Yildirim T, Akkuzu B, Yilmaz I. Comparison of nasal region dimensions in bilateral choanal atresia patients and normal controls: a computed tomographic analysis with clinical implications. Int J Pediatr Otorhinolaryngol 2009; 73: 329-335.

8. Burrow TA, Saal HM, de Alarcon A, Martin LJ, Cotton RT, Hopkin RJ. Characterization of congenital anomalies in individuals with choanal atresia. Arch Otolaryngol Head Neck Surg 2009; 135: 543-547.

9. Uzomefuna V, Glynn F, Al-Omari B, Hone S, Russell J. Transnasal endoscopic repair of choanal atresia in a tertiary care centre: a review of outcomes. Int J Pediatr Otorhinolaryngol 2012; 76: 613-617.

10. Teissier N, Kaguelidou F, Couloigner V, Francois M, Van Den Abbeele T. Predictive factors for success after transnasal endoscopic treatment of choanal atresia. Arch Otolaryngol Head Neck Surg 2008; 134: 57-61.

11. Warner D, Brietzke SE. Mitomycin C and airway surgery: how well does it work? Otolaryngol Head Neck Surg 2008; 138: 700-709.

12. Hengerer AS, Brickman TM, Jeyakumar A. Choanal atresia: embryologic analysis and evolution of treatment, a 30-year experience. Laryngoscope 2008; 118: 862-866.

13. Schoem SR. Transnasal endoscopic repair of choanal atresia: why stent? Otolaryngol Head Neck Surg 2004; 131: 362-366.

14. Kim H, Park JH, Chung $H$, et al. Clinical features and surgical outcomes of congenital choanal atresia: factors influencing success from 20-year review in an institute. Am J Otolaryngol 2012; 33: 308-312.

15. Van Den Abbeele T, Francois M, Narcy P. Transnasal endoscopic treatment of choanal atresia without prolonged stenting. Arch Otolaryngol Head Neck Surg 2002; 128: 936-940.

16. Bozkurt MK, Keles B, Azimov A, Ozturk K, Arbag $\mathrm{H}$. The use of adjunctive topical mitomycin in endoscopic congenital choanal atresia repair. Int J Pediatr Otorhinolaryngol 2010; 74: 733-736.

17. Holland BW, McGuirt WF Jr. Surgical management of choanal atresia: improved outcome using mitomycin. Arch Otolaryngol Head Neck Surg 2001; 127: 1375-1380.

18. Prasad M, Ward RF, April MM, Bent JP, Froehlich P. Topical mitomycin as an adjunct to choanal atresia repair. Arch Otolaryngol Head Neck Surg 2002; 128: 398-400.

19. Al-Ammar AY. Effect of use of mitomycin C on the outcome of Choanal atresia repair. Saudi Med J 2007; 28: 1537-1540. 